\title{
BIM and IoT: A Synopsis from GIS Perspective
}

\author{
U. Isikdag a \\ ${ }^{a}$ Mimar Sinan Fine Arts University, Department of Informatics, Istanbul, Turkey - uisikdag@ gmail.com
}

\section{Commission II, WG II}

KEY WORDS: BIM, IoT, GIS, Integration, Sensors

\begin{abstract}
Internet-of-Things (IoT) focuses on enabling communication between all devices, things that are existent in real life or that are virtual. Building Information Models (BIMs) and Building Information Modelling is a hype that has been the buzzword of the construction industry for last 15 years. BIMs emerged as a result of a push by the software companies, to tackle the problems of inefficient information exchange between different software and to enable true interoperability. In BIM approach most up-to-date an accurate models of a building are stored in shared central databases during the design and the construction of a project and at postconstruction stages. GIS based city monitoring / city management applications require the fusion of information acquired from multiple resources, BIMs, City Models and Sensors. This paper focuses on providing a method for facilitating the GIS based fusion of information residing in digital building "Models" and information acquired from the city objects i.e. "Things". Once this information fusion is accomplished, many fields ranging from Emergency Response, Urban Surveillance, Urban Monitoring to Smart Buildings will have potential benefits.
\end{abstract}

\section{INTRODUCTION}

\subsection{The IoT Paradigm}

Internet-of-Things (IoT) focuses on enabling communication between all devices, things that are existent in real life or that are virtual. Being a real thing or being a virtual thing is not the focus of the IoT approach. IoT approach concentrates on the state of "Things" and on informing "Things" about other "Things". The IoT aims to make "Things" producers or consumers of information. An IoT environment is analogous to a nervous system in terms of exchange of information. In every state change, a "Thing" provides information about its new state. For example, in a house that is realizing the IoT paradigm a window will mention that it is open at this current moment, or a door will mention that it is locked at this current moment. On the other hand the IoT paradigm suggests that the "Things" needs to become clever enough to take actions in reaction to the messages coming from humans or in reaction to state changes in other objects. For instance a Human-to-Thing interaction can be initiated as a result of a cell-phone message (SMS) e.g. for turning on the heater before coming to home, or turning off the lights 30 mins. after leaving the office. The IoT era is starting and will change our lives in a dramatic way.

\subsection{Internet as a Utility \& Web Services}

It has been around 20 years since we have met with the concept of the Internet and WWW. First Internet was interpreted as a new method of talking with friends, a new method of sending mail, a new approach to marketing, and sales of goods and services. The technologies that has come to our lives with internet have significantly changed and transformed over the 20 years, although there still exists the issue of digital divide. For example, mobile phone technology has been developed and transformed rapidly. Today cell phones are only fully-functional when they are connected to the internet. The situation is same for tablet computers, a similar technology that emerged in last 10-15 years and transformed rapidly to benefit most from the opportunities provided by the internet. How can we interpret this situation to depict the role of a single technology, such as internet, when a "Thing" become useless if it does not benefit from a certain technology? We can build up the analogy of electricity/water and dishwashers. In that situation "Things" (i.e. the machine) need to benefit from the utilities (e.g. water) in order to work properly. Once a technology need comes to a level that a "Thing" would not work properly without that technology, that technology is started to be termed -not any more as a technology- but as a utility. The situation is becoming the same today for the internet. The internet is becoming the key utility of the future.

From this perspective the Web Services can be thought as interaction endpoints of this utility (i.e. Internet). A dominant architectural approach for interacting with web endpoints (i.e. web services) today is Representational State Transfer (REST). The approach enables the users to interact with components/services that are being provided over the internet. Among with Web Services, there are other options for interaction. For instance human-to-thing or thing-to-thing interaction over the internet can also be enabled and facilitated by sending messages. These messages can be sent on demand by the humans or when a state change occurs in a hard/soft (real/virtual) web component. Message Brokers are middleware tools that helps us to distribute these messages.

\subsection{A Prologue to BIM}

Building Information Models (BIMs) and Building Information Modelling is a hype that has been the buzzword of the construction industry for last 15 years. BIMs emerged as a result of a push by the software companies, to tackle the problems of inefficient information exchange between different software and to enable true interoperability. IFC is a universally 
recognised BIM schema (and information exchange) standard which is developed to facilitate information exchange between construction industry applications. The BIM (i.e. model) servers / BIM data stores are in use together with IFC models to enable real-time design information exchange and sharing. During the design and the construction of a project and at post-construction stages most up-to-date an accurate models of a building are stored in shared central databases. This has opened new doors. Industry started to focus on making pre-construction simulations using these models which has been accompanied by multiple stakeholders, which is now termed as the $\mathrm{nD}$ modelling approach. Later, the information residing in the models has been maintained following the construction phase, and the models started to act as virtual ID cards of the buildings.

\subsection{Building Representations of Digital City Models}

Today city modelling standards such as CityGML provides representation of detailed level of building information, also including building interiors. For instance a CityGML Model of Level of Detail (LOD) 4 will provide detailed information about buildings` interior elements. The building representations in city models utilize information transferred from BIM to represent the semantics of building, while real time information regarding indoors needs to be provided by sensors at IoT nodes. Thus as a city can be regarded as a living eco-system, most accurate city information models will utilize information coming from both i.) building representations in city models and BIMs and ii.) real and virtual sensors.

In another approach of information representation, it is also possible that every object in the city and every object in a building (i.e. regardless of being a building component or a furniture or a device) can be represented in form of a virtual sensor or can be associated (1-to-1 or 1-to-N) with virtual sensors.

\subsection{Focus of the paper}

GIS based city monitoring / city management applications require the fusion of information acquired from multiple resources, Things, Models, Virtual Objects, Real Objects. This paper focuses on providing a method for facilitating the GIS based fusion of information residing in digital building "Models" and information acquired from the "Things". Once this information fusion is accomplished, many fields ranging from Emergency Response, Urban Surveillance, Urban Monitoring to Smart Buildings will benefit from this fusion. Following this section, section 2 provides information on Building Information Modeling, section 3 focuses on Internet of Things, section 4 elaborates on RESTful Web Services, while section 5 provides a RESTful design pattern for integration of GIS-BIM and IoT Nodes (i.e. sensors).

\section{BUILDING INFORMATION MODELLING}

BIM acronym is used to denote two concepts Building Information Model (i.e. a shared information model) and Building Information Modeling (i.e. an information management methodology). BIM currently is the most active field of Construction Informatics research globally. Building Information Modeling is a methodology that addresses the problems related to sharing of information, interoperability and efficient collaboration throughout the lifecycle of a building (i.e. from feasibility and conceptual design through to demolition and re-cycling stages). This well-known methodology has origins in two distinct but related fields. The first one is Computer Aided Design (CAD), the latter one is Representation of Building Product Information -in an organised manner-. Building Information Modelling has been realised as a process of managing a project through a single shared information backbone (which is formed by shared information models i.e. BIMs). The models also play the role of interoperability enabler in this case. Construction process simulations can now be efficiently prepared and managed using Building Information Models, time and resource management can be completed more efficiently, clashes between elements can be identified. Building costs can be calculated automatically as a result of automatic quantity take-offs. Countries such as Singapore use BIMs to validate that building models are compliant with code and regulations. BIM approach plays a key role in the design and management of energy-efficient buildings.

Depending on the information requirements Building Information Models can have different functions such as being a Space Linker that links macro and micro urban spaces(i.e. city and building level), an Interoperability Enabler which facilitates information sharing between various stakeholders and the software applications they use, a Data Store which stores the building information throughout the lifecycle of a building, a Procurement Facilitator that facilitates several procurement related tasks in the building lifecycle, a Collaboration Supporter through enabling the use and management of shared building information in real-time, a Process Simulator by facilitating the simulation of construction processes (i.e. nD), a System Integrator which enables the integration of several information systems across the industry, a Building Information Service which can serve real-time on-demand building information over the Internet, a Green Builder that enables advanced analysis supporting the design and construction of environment friendly/energy efficient buildings, and a Life Saver which facilitates emergency response operations (Isikdag and Underwood, 2010).

The commonly known schema standards in the field of BIM are Industry Foundation Classes (IFC) and the CIMSteel Integration Standards (CIS/2), both of which are defined using STEP (ISO10303) description methods. IFC is the most popular BIM standard (and schema) today. IFC aims to improve the communication, productivity, delivery time, cost, and quality throughout the design, construction and maintenance of buildings. An IFC model consists of classes (i.e. data objects in runtime) to describe building elements and construction process details. IFC allows sharing and exchange of building product and construction process related information among the stakeholders of a project. In a BIM based project management and delivery effort, each specific discipline can contribute to the development of the model during the design process and can output an own (i.e. HVAC related, Electricity related...) view of the model. IFC became an ISO Publicly Available Specification (as ISO 16739) in 2005. Currently most CAD, structural analysis, time/cost management, FM software are capable of importing and exporting IFC files, and some software are capable of acquiring information from an IFC model through the use of a shared resource such as a model server database. The model views that can be regarded as model subsets can be generated from BIM files or BIM databases or BIM servers by using APIs, and web interfaces of the servers. The views that are generated can be transient (i.e. temporary) or persistent (i.e. permanent), the generation of the both type of views is possible depending on the needs of the project. Model mapping and derivation approaches are used to generate these subsets (i.e. the model views.). In this derivation process the original model is called as the base model and the derived 
model is called as the model view. The developer and current supporter of the IFC standard Building Smart (i.e. formerly International Alliance for Interoperability) today views the role of BIMs, in three dimensions, as terminology, processes and data. It is indicated that if all these three dimensions can be defined and implemented accurately and information representation is accomplished successfully in these directions and if efficient communication would be established between them, the industry will very much benefit from better integration in construction projects. In order accomplish these aims BuildingSmart developed three international standards, Data Dictionary Standard - International Framework for Dictionaries (IFD), process Definition Standard - Information Delivery Manual (IDM) and Data Modelling Standard -Industry Foundation Classes (IFC). There also exists tools such as MVD (Model View Definitions) which provide definitions of Views for specific aspects such as Architectural Design, Structural Analysis, and HVAC design in order to support BIM based construction management practices.

A recent development in the field has been the adoption of Open BIM Collaboration Format (BCF) as a BuildingSmart standard. The BCF is an open format which aims to support the workflow communication during the BIM based construction management processes.

The BIM approach when it has come into the focus of the construction industry was viewed as a strategy to use shared information models. Now the approach has been evolved into an information management strategy which mainly supports the design process but also facilitates the processes during the construction stages and during the use of the building. Currently BIM is also viewed as a construction management and an integrated project delivery (IPD) method.

The evolution of BIM from a shared information model or from a schema standard for defining information models to an information and construction management methodology is very fast and noticeable. In today's world BIM based construction management and project delivery methodology is required by many owners of the large facilities such as airports, shopping malls etc. Today several projects in U.S, Singapore, Dubai/Middle East, and Europe require, the involvement of BIM Managers (a profession which is emerged in last 10 years) and BIM based project delivery.

The new BIM based project delivery requirement, overloads the model (i.e. that is defined as BIM or BIM 1.0). Thus a transformation from BIM 1.0 to BIM 2.0 is required. The transformation of BIM from BIM 1.0 to BIM 2.0 can be investigated in two dimensions. The first one is changing role of information models from being a shared information resource to something more complicated. The second dimensions is the emerging role of BIM as a new construction management method. Underwood and Isikdag (2011) stated that 4 newly emerging dimensions in management of building information towards transforming from BIM to BIM 2.0 focuses on enabling an i.) integrated environment of ii.) distributed information which is always iii.) up-to-date and open for iv.) derivation of new information. Various information technologies such as cloud computing, sensor networks, stateless web services and semantic web will contribute to this new definition of BIM.

- Cloud Computing: The term refers to the use of Internet (i.e. the cloud) for computation, but its context is very broad. For instance the term today refers to managing highly scalable and customizable virtual hardware and software resources (which are provided as services)." Cloud computing today is broken down into three segments as providing "Software as a Service
(SaaS)", "Platform as a Service (PaaS)" and "Infrastructure as a Service (IaaS)". The cloud computing is also closely related with virtualization and involves the virtualization of data centres and data warehouses. The BIM based project delivery will benefit from the use of Software as a Service approach, i.e. by making concurrent use of cloud based design, analysis, time/cost management software. In addition, cloud based BIM databases will facilitate the sharing of information between the members of a project team. These cloud based BIM databases will provide data objects representing each building element and process/task which will reside in virtualized data centres. By this way, the project team will have an access to the BIM components (i.e. data objects) over the cloud and this will minimise the storage and sharing related problems. The BIM 2.0 components can be regarded as virtual building elements which real time data can be attached to. Attachment of real-time information coming from sensors will provide a world of new opportunities for the construction and urban management domains.

- Real-Time Information: Recent developments in the field of BIM has demonstrated that Building Information Models become considerably successful in representing semantic information (such as material of a wall, type of windows etc.) along with their most detailed geometric representation. Even though the semantic information in the models is represented with great success, at the post-construction/operation stage model itself becomes stateless, which means that it does not represent the building as a living entity, but it only represents the as-built or as-operated form of the building. This results in a situation that BIM remains as -not more than- a usage manual for the building. For example by querying a BIM one can find out whether a door in a building is constructed of timber, or if a door has been constructed (or not) on a given date, or can query changes made on that door until today, but it is not possible to get informed on whether that door is open or closed at a certain point in time by using BIMs or BIM-based information infrastructures. In order to make BIM a living information resource, i.e. an information provision model, information coming from IoT (Internet of Things) nodes and sensors/sensor networks needs to be integrated with the BIM 2.0. The sensors that are monitoring every building element (when integrated with data objects of the BIM) will provide meaningful information about the states of the building elements and also regarding the states of the spaces (rooms, corridors etc.) in the building. Several conditions in rooms such as temperature, gas levels, sound levels, existence of people and animals, air pollution rate, humidity can be observed and this information can be provided in real time over the cloud. Furthermore state of doors and windows (i.e. such as being open/closed), conditions of different systems working within a building/facility such as situation of elevators and escalators can be easily monitored in real time over the internet by the implementation of BIM 2.0. In order to manage this monitoring process efficiently, web services (and especially the RESTful approach) forms an excellent architectural framework. 
- RESTful Web Services: Web services can be regarded as public interfaces to software components which make these components accessible over the web. It is possible to interact with web services in a very similar way to interaction with APIs. RESTful Web Services is an architectural approach that uses HTTP protocol methods to invoke the methods in web service (interfaces). Create/Read/Update/Delete (CRUD) operation set can be performed over the BIM objects using RESTful architectures and this brings great flexibility in management of shared BIMs. BIM updates through web services by multiple users results in up-to-date and highly accurate BIMs that are reflecting the real-time situation of the construction or building in use. Information residing in BIMs and feeds published by IoT nodes can be integrated in a loosely couple way using RESTful web services to generate mash-ups which represent real time information about buildings and city models.

- Big Data: Big data structured with well-defined ontologies offers unique opportunities for city management applications. If information derived from multiple resources (buildings, roads, electrical appliances, urban furniture...) in a city model can be organized in compliance with city modelling standards and well-defined ontologies, semantic queries such as "Would you provide me the number of working elevators and escalators in the Petronas Towers between 12.00-14.00?", "Would you provide me the average $\mathrm{CO} 2$ level in top 20 floors of 5 of the highest buildings in Johor Bahru?" or "Please provide me the difference between temperatures in my hotel room in Kuala Lumpur, and my office in Istanbul." can be responded. The success rate is directly proportional with the level of integration of city information coming from multiple resources including buildings and other city objects.

\section{INTERNET OF THINGS}

Today the role of Internet is evolving from being a communication medium for people to being a communication medium between people, between people and devices, and between devices. The Internet of Things (IoT) approach defines a global network structure based on standards and set of communication protocols where physical and virtual devices (i.e. known as Things) communicate with each other, publish, consume, exchange and share information in real time. IoT implementation enables "Things" (i.e. devices, city objects, building elements...) to communicate with each other and this provides unique opportunities for the development of smart buildings and smart cities. In smart buildings which implement the IoT approach, a door would have the ability to connect with the fire alarm, or a chair would communicate with indoor lights. In smart cities, a car would communicate with the parking space, a train can communicate with other trains, a bus can communicate with a bus stop.... The list can be extended. Today can be regarded as the start of the Internet of Things era. Internet of Things covers the utilisation of sensors and near field communication technologies such as RFID or NFC, together with information provision through embedded systems. The devices/tools that take part in an IoT environment can range from cell phones to RFID readers, GPS devices to tablets, embedded control systems in cars to weather stations. Single Board Computers -SBCs- (which are economically feasible and easy to program) today are playing a vital role for development and testing of many IoT related technologies. Although, Single Board Computers are devices that are developed as a proof of concept and experimental tools they have an ability to acquire information from its surroundings by sensors, either embedded in them / or connected to them. The SBCs consist of a single circuit board memory and a microprocessor/microcontroller. Most of these SBCs have I/O interfaces where many kinds of different sensors can be plugged in. The processors of the SBCs are usually of low cost. The size of devices are small and is equal to the size of a matchbox or a playing card. Depending on the type of the computer it is possible to run the versions of Embedded Linux and even Windows, while some only have programmable microprocessors/microcontrollers which only provide output to their proprietary workbench. Today SBCs can be used as small size web servers, grid computing components, and most importantly as nodes of an IoT architecture. There have been more than 20 different types of SBCs produced by different vendors until today, and they now act as the foundational hardware layer for the development of IoT concepts and IoT software layers.

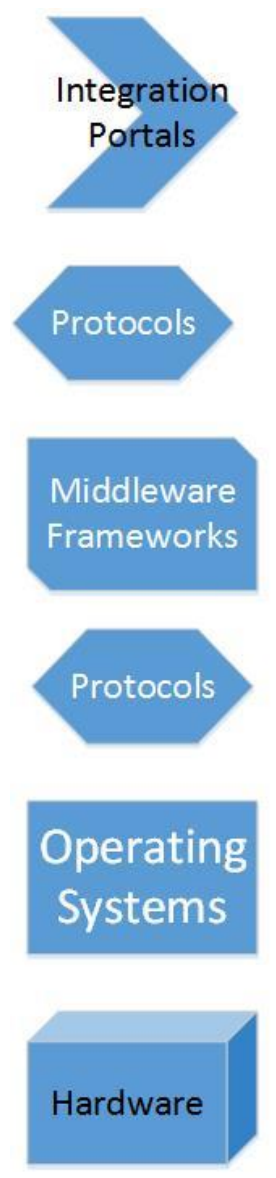

Figure 1.Components of an IoT architecture

As Figure 1 illustrates an IoT architecture consists of several software layers. The IoT hardware forms the foundational layer of the architecture. The first software layer is formed by the operating system that is installed to operate the hardware components. The hardware components can either be an embedded system or a SBC where the hardware would mostly controlled by an operating system. There are exceptions to this situation for SBCs such as Arduino (Arduino Web Site, 2015), where the hardware directly communicates with the middleware 
or a programming framework. Some examples of the IoT operating systems include, OpenWRT (OpenWRT Web Site, 2015), Raspbian (Raspbian Web Site, 2015), Contiki OS (Contiki Web Site, 2015). The protocols play a role in enabling communication between different software layers. When the embedded system or a SBCs runs an OS, communication protocols enable the communication between the middleware tier and the OS. In addition, protocols also facilitate communication between the middleware (or development) frameworks and integration portals.

Physical layer protocol examples include IEEE 802.15.4, Zigbee, Bluetooth and NFC. The TCP/IP protocol stack provides support for the enablement of IoT, but in fact, in an IoT network architecture there are other unique protocols that specifically focus on enabling and facilitating the communication. Protocols such as RPL and 6LowPAN facilitate the communication at network layer, where COAP is at application layer and is specifically defined for supporting machine to machine communication. There are also other protocols that are benefiting from TCP/IP application and transport layers for enabling message exchange between devices such as MQTT and XMPP.

The middleware and development frameworks usually forms the core layer of the IoT software. Middleware (or development) frameworks act as a bridge between devices i.)to enable Machine-to-Machine (M2M) communication, or ii.)to facilitate provision of the information acquired from the surrounding environment either to the users directly or to the integration portals. This layer mainly focuses on orchestrating the exchange of information and interactions between i.)devices, and also ii.)between devices and other software layers. The IoT frameworks can have many functions ranging from providing development / coding environment to hardware, to providing component containers to interact with real or simulated (virtual) sensors. Some examples of the middleware are AllJoyn (AllSeen Alliance Web Site, 2015) and Eclipse IoT (Eclipse IOT Website, 2015), also some hardware vendors such as Arduino provide their own development environment / framework.

An integration portal can be defined as the software components where information acquired from real or virtual sensors is combined, reorganized and presented to the end-users (e.g. to citizens of a city for a city management IoT architecture). The integration portals provide means for facilitation of machine-tomachine (M2M) communication and information exchange, but their main role is to enable the visualisation of information collected from multiple resources and provision of information to web interfaces for interaction with the IoT nodes (i.e. with sensors and actuators connected to them). Among well-known examples of these portals are Xively (Xively, 2015), and Paraimpu (Paraimpu Web Site, 2015).

\section{RESTFUL WEB SERVICE ARCHITECTURES}

As indicated in the introduction section Web Services can be regarded as interaction endpoints of Internet. A user or a software component can utilize Internet as a medium to interact with other software components when they provide Web Service endpoints. REST (REpresentational State Transfer) architectural approach suggests that, a web service can be built upon resources (i.e. anything that is available digitally over the web), their names (identified by uniform indicators, i.e. URIs) representations (i.e. metadata/data on the current state of the resource) and links between the representations. REST's decoupled and easy to use style made REST a popular architecture for cloud-based APIs, such as those provided by
Amazon, Microsoft, and Google. When software components use RESTful architecture principles for providing web interfaces, they are called RESTful APIs (Application Programming Interfaces) or REST APIs. As REST supports stateless existence of services where the server does not store the number or state of the clients, REST is very efficient for the server side. One drawback is the number of operations on resources is limited with the methods provided by the HTTP protocol. GET, PUT, POST and DELETE are most commonly used HTTP methods in RESTful architectures. On the other hand, the hierarchical naming convention used by most REST APIs provides a flexibility where each RESOURCE is identified by a Universal Resource Identifier (URI) which is defined by nouns. For example a request that is using hierarchical naming convention mechanism such as "HTTP GET http://www.mybuilding.com/floor/1/flat/22/room/2/lights/situati on" can provide a meaningful response. Another example for the use of this convention can be given as "HTTP GET http://www.mybuilding.com/floor/1/flat/2/room/2/lights/turn/on ". The RESTful APIs provide the advantage of exchanging plain text messages, messages in XML or JSON formats which are easy to interpret and comply with. As mentioned earlier this paper focuses on providing a method for facilitating the GIS facilitated fusion of information residing in digital building "Models" and information acquired from the "Things". In parallel with the focus of the paper next section presents a design pattern that is using RESTful approach to integrate GIS, information acquired from BIMs and information acquired from the IoT nodes. Once this integration is accomplished many fields ranging from Emergency Response, Urban Surveillance, Urban Monitoring to Smart Buildings will benefit from this integration and information fusion.

\section{A DESIGN PATTERN PROPOSAL ON INTEGRATION OF GIS-BIM AND IOT NODES}

The design pattern proposal provided here focuses on providing an architecture for integration of information coming from BIM Objects and IoT Nodes. The role of GIS is defined as an integration platform, as well as a visualisation platform for integrated information. The pattern is illustrated in Figure 2.

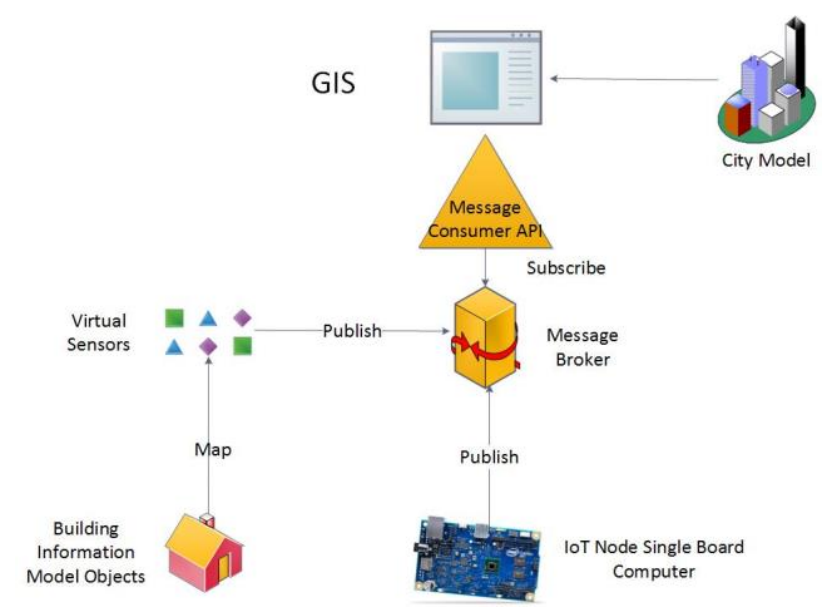

Figure 2: GIS-BIM-IoT Nodes Integration Pattern

The data layer of the architecture consists of BIM Objects that are residing in a BIM database, BIM server or a BIM file for representing the buildings. Geometric and semantic information about other city objects will be acquired from a digital city model which can be directly imported into a GIS. It is assumed 
that BIM in this pattern is BIM 2.0 model which is capable of representing information regarding the states of the building elements (such as being on for a light, being open/closed for a door etc.). Every BIM object (i.e. representing beam, column, space etc.) can be mapped into virtual sensors that can provide information whenever a state change occurs at BIM objects (e.g. when an elevator stops working). The virtual BIM sensors in this pattern will provide geometric and semantic information together with information related to the state of the building elements. As these virtual sensors will also provide information about building geometries and semantics, this information can be used to represent the buildings within a GIS environment. The state information regarding other city objects (which are residing in GIS) will be provided by real sensors that are connected to SBCs (IoT nodes). Virtual and real sensors are used side-to-side in this pattern to publish (geometric/semantic/ and state related) information in form of messages broadcasted whenever a state change occurs at a building element (a person leaves a house and closes the main door) or at a city object (e.g. when a bus arrives to a bus stop near that house). A message broker forms the middleware tier of the pattern which acts as a listener for these messages. Once the message broker receives messages from real and virtual sensors it broadcasts them to its subscribers. The first set of messages in the broadcast regarding a building will be providing the geometric/semantic information about building elements. A RESTful message consumer API that is coupled with a GIS will be the subscriber of the Message Broker and will get informed whenever a state change occurs at a building element or at a city object. The API can then be queried at certain intervals or send real-time information into the city model by interaction with GIS APIs. The real-time model will than be visualised within a GIS environment. The GIS environment can be in form of a standard desktop GIS or a web based GIS.

\section{CONCLUSIONS}

The proposed pattern will provide various advantages that can be summarized as below;

GIS Type Independence: The proposed architecture can be realized by using any GIS client that can either be an off-theshelf desktop GIS, a GIS server or a web GIS implementation. Only requirement from the client side is the ability to communicate with a service consumer API. The loosely coupled nature of the proposed architecture would bridge the key technologies for acquiring and presenting real-time building information and information acquired from sensors. The building information gathered from the virtual sensors such as indoor temperature, humidity, state of building elements (i.e. the window being open/closed), the state of elevators and escalators (i.e. in operation or not), indoor oxygen and other gas levels will be available for the interested parties regardless of their hardware, operating system and software they use. Similarly, information gathered from city objects such as air pollution, sound levels, solar radiation levels, parking space occupancies, bus locations, underground locations, will be available in real time.

24/7 Monitoring of the City and Buildings: City Dashboard applications would be the main consumers of this ubiquitous information. By the implementation of the described architectures, the information that is provided by the BIMs and IoT Nodes, will be available without interruption even when an emergency situation or disaster occurs. In this case information coming from building models information provides advantages in answering the questions such as "Would you provide the average $\mathrm{CO} 2$ level in the rooms which are not affected by the fire?", "Would you provide the number of doors which are open in the floors that are affected by the flood?" and so on, can be answered in real time. Furthermore, for example information coming from IoT nodes can provide information on the location of emergency response vehicles.

Crowd-Sourced Monitoring: This approach is extremely useful to enable ubiquitous monitoring of emergency/disaster situation. Crowd-Sourced Monitoring can be very useful in realtime monitoring of the spreading of the fire in a forest, i.e. IoT Nodes located in the buildings in the forest area can provide information on if the fire has reached the region by providing temperature and gas level information inside and outside the building. Crowd-Sourced Monitoring can also be useful in Tsunami events, lots of houses can become flooded in a very short time period, and a crowd-of-nodes that are still in operation (near buildings that are not flooded) would provide key information regarding the invasion of flood water within the region.

Further research will focus on the implementation of this software architecture and its testing with real life case studies.

\section{REFERENCES}

Arduino Web Site (2015) Available online at: www.arduino.cc

Isikdag, U., Underwood, J. A Synopsis of the Handbook of Research on Building Information Modelling, CIB World Congress, Salford, UK, May 10-13, 2010.

Underwood, J., Isikdag, U. (2011) "Emerging technologies for BIM 2.0", Construction Innovation, Vol. 11 Iss: 3, pp.252 258

Contiki Web Site (2015) Available online at: http://www.contiki-os.org

OpenWRT Web Site (2015) Available online at: https://openwrt.org

Raspbian Web Site (2015) Available online at: http://www.raspbian.org

AllSeen Alliance Web Site, 2015 Available online at: https://allseenalliance.org

Eclipse IOT Website (2015) Available online at: http://iot.eclipse.org

Xively (2015) Available online at: http://www.xively.com

Paraimpu (2015) Available online at: https://www.paraimpu.com 